\title{
Boosting Influences From circ_0048856 On Non- Small Cell Lung Cancer Advancement Via Aiming At miR-1287-5p
}

\section{Yinzai He}

Inner Mongolia People's Hospital

Yanheng Liu

Inner Mongolia People's Hospital

Nier Cha

Inner Mongolia People's Hospital

Yanwei Gao

Inner Mongolia People's Hospital

\section{Feng Li}

Inner Mongolia People's Hospital

Meng Zhang

Inner Mongolia People's Hospital

Wei Luan ( $\square$ okdhnd@126.com )

Inner Mongolia People's Hospital https://orcid.org/0000-0002-7327-7567

Primary research

Keywords: Circ_0048856, MiR-1287-5p, NSCLC, Prognostic

Posted Date: April 12th, 2021

DOl: https://doi.org/10.21203/rs.3.rs-335806/v1

License: (9) This work is licensed under a Creative Commons Attribution 4.0 International License.

Read Full License 


\section{Abstract}

Background: Non small cell lung cancer (NSCLC) is one of the main causes of death in human diseases. In this study, we aimed to explore the prognostic value of serum circ_0048856 in patients with NSCLC and its relationship with $m i R-1287-5 p$.

Methods: The expression levels of circ_0048856 and miR-1287-5p in serum of NSCLC patients and healthy people were detected by qRT-PCR. The relationships between circ_0048856 expression and clinical data were evaluated via $\chi^{2}$ test. Overall survival analysis was carried out via Kaplan-Meier method with log rank test. Cox regression analysis was taken to evaluate the prognostic ability of circ_0048856 in NSCLC. Luciferase reporter gene was used to detect the targeting relationship between circ_0048856 and miR-1287-5p.

Results: The expression of circ_0048856 in serum of NSCLC patients was significantly higher than that of healthy volunteers $(P<0.001)$. And its high expression was significantly correlated with TNM stage $(P=$ $0.002)$ and lymph node metastasis $(P<0.001)$. In addition, Kaplan Meier log rank test analysis showed that the overall survival of patients with high expression of circ_0048856 was significantly shorter than that of patients with low expression of circ_0048856 (log rank test, $P=0.002$ ). Serum circ_0048856 expression is an independent prognostic biomarker in NSCLC patients. Furthermore, we found that $m i R$ $1287-5 p$ is a direct downstream miRNA of circ_0048856 through luciferase reporter gene analysis, and there is a negative correlation between circ_0048856 and $m i R-1287-5 p$.

Conclusion: Serum circ_0048856 is an independent prognostic biomarker in patients with NSCLC. Circ_0048856 can participate in the progression of NSCLC by targeting miR-1287-5p.

\section{Background}

Lung cancer is a highly heterogeneous cancer [1]. Every year, about 1.8 million people are diagnosed with lung cancer and 1.6 million people die of lung cancer. The 5-year survival rate is very low, which is usually between $4-17 \%$ due to the stage and regional differences [2]. Lung cancer mainly includes non-small cell lung cancer (NSCLC) and small cell lung cancer (SCLC), accounting for $76 \%$ and $13 \%$ respectively [3]. Smoking is a major risk factor for lung cancer. Of course, the occurrence of lung cancer is also related to environmental and genetic factors [4,5]. At present, surgical treatment is still the best treatment for early lung cancer [6]. In recent years, with the progress of medical technology, the treatment methods of NSCLC are diverse, and the mortality has been reduced [7]. However, NSCLC patients are prone to metastasis. Metastatic NSCLC is an advanced tumor that is difficult to cure, with low survival rate, almost no cure possibility and poor prognosis [8]. Therefore, it is urgent to find new prognostic markers and improve the treatment level of NSCLC.

Circular RNAs (circRNAs) are a class of covalently closed RNA loops widely expressed in eukaryotic cells. They are produced by a process called reverse splicing, and they are evolutionarily conserved $[9,10]$. Studies have shown that circRNAs are microRNA (miRNA) sponges, protein regulators, transcription 
regulators, protein templates, etc [11]. CircRNAs have a variety of biological functions and participate in a variety of physiological and pathological processes, including tumorigenesis and development [12-14]. The emergence of the circRNAs family provides a new entry point for the research and treatment of tumors. Studies have found that many circRNAs are involved in the progress of NSCLC. For example, Xu et al. pointed out that circ_0001869 promotes the progress of NSCLC through sponge miR-638 and promoting the expression of FOSL2 [15]. Sun and colleagues found circ_0000376 is highly expressed in NSCLC clinical specimens, which is related to adverse pathological parameters, and it can significantly promote the proliferation and metastasis of NSCLC cells, and enhance the drug resistance of cells [16]. Among them, circ_ 0048856 is up-regulated in NSCLC samples, which may participate in the occurrence and development of NSCLC as a cancer promoting factor [17]. However, the prognostic value and molecular mechanism of circ_0048856 in NSCLC are still unclear. Therefore, the study of serum circ_0048856 as a prognostic marker of NSCLC has important clinical significance.

This study examined the prognostic significance of circ_0048856 in serum of patients with NSCLC. Further verification found that circ_0048856 and miR-1287-5p have a targeted relationship, and found that there is a negative correlation between them, which provides a new theoretical basis for the treatment of patients.

\section{Methods}

\section{Subjects and samples}

A total of 121 cases pathologically diagnosed with NSCLC and 113 healthy individuals were collected in this study. Before sampling, they did not receive any treatment including radiotherapy or chemotherapy. $5 \mathrm{~mL}$ fasting blood specimen were obtained from all subjects, then serums were separated from whole blood via centrifugation. The supernate was immediately stored at $-80^{\circ} \mathrm{C}$ until for use. All patients were followed up by telephone for 5 years. The clinicopathological features of the cases were shown in Table 1. This study was approved by the Ethic committee of Inner Mongolia People's Hospital. The written informed consent forms were provided from each participant in the present study prior to sampling.

Table 1. Relationship between circ_0048856 expression and clinical features in NSCLC 


\begin{tabular}{|c|c|c|c|c|}
\hline \multirow[t]{2}{*}{ Clinical Features } & \multirow[t]{2}{*}{ Cases $(n=121)$} & \multicolumn{2}{|c|}{ circ_0048856 expression } & \multirow[t]{2}{*}{$P$} \\
\hline & & Low $(n=58)$ & High $(n=63)$ & \\
\hline Age (years) & & & & 0.446 \\
\hline$\leq 60$ & 52 & 27 & 25 & \\
\hline$>60$ & 69 & 31 & 38 & \\
\hline Gender & & & & 0.275 \\
\hline Male & 83 & 37 & 46 & \\
\hline Female & 38 & 21 & 17 & \\
\hline Smoking history & & & & 0.269 \\
\hline No & 46 & 25 & 21 & \\
\hline Yes & 75 & 33 & 42 & \\
\hline Tumor size(cm) & & & & 0.322 \\
\hline$\leq 3$ & 59 & 31 & 28 & \\
\hline$>3$ & 62 & 27 & 35 & \\
\hline TNM stage & & & & 0.002 \\
\hline $\mid-I I$ & 47 & 31 & 16 & \\
\hline III-IV & 74 & 27 & 47 & \\
\hline Lymph node metastasis & & & & $<0.001$ \\
\hline No & 79 & 48 & 31 & \\
\hline Yes & 42 & 10 & 32 & \\
\hline Differentiation & & & & 0.274 \\
\hline Poor & 81 & 36 & 45 & \\
\hline Well/moderate & 40 & 22 & 18 & \\
\hline
\end{tabular}

\section{Cell culture}

NSCLC cell lines A549 and H1299, as well as human bronchial epithelial cell (HBE) were purchased from American Type Culture Collection (ATCC). The cells were cultured in DMEM medium containing $10 \%$ fetal bovine serum in $37{ }^{\circ} \mathrm{C}$ and $5 \% \mathrm{CO}_{2}$ saturated humidity incubator for subculture once every two days. When the cells were covered with the bottom of the culture bottle and fused into a single layer, and the 
density reached $70 \% \sim 80 \%$ fusion, the cells were digested with $0.25 \%$ trypsin and $1: 3$ ratio is used for subculture.

\section{qRT-PCR}

RNA Isolation Kit (Qiagen, Hilden, Germany) was used to isolate total RNA based on operation manual. The qualitative integrity of purified total RNA was tested by capillary electrophoresis using 2100 Bioanalyzer (Agilent Technologies, Palo Alto, CA). RevertAid ${ }^{\mathrm{TM}}$ First Strand cDNA Synthesis Kit (Ferments, Vilnius, Lithuania) was adopted to perform the reverse transcription. The expression of gene in specimens were measured via quantitative real-time PCR (qRT-PCR). The reaction was carried out with SYBR Premix Ex Taq ${ }^{\text {TM }}$ (TaKaRa, Dalian, China) kit in ABI 7500 instrument (ABI). The experiment was repeated at least 3 times. Finally, the levels of gene in NSCLC patients or healthy control group were calculated via the $2^{-\Delta \Delta C t}$ method.

\section{Cell transfection}

Cell lines in exponential growth period were conducted cell transfection. The transfection vectors were sicirc_0048856 and si-NC were purchased from Guangzhou RiboBio Co., Ltd.,(Guangzhou, China). They were transfected into NSCLC cells by LipofectamineTM2000 (Invitrogen, CA, USA) following the instruction of manufacturer, then cultured in $37^{\circ} \mathrm{Chumididied} \mathrm{atmosphere} \mathrm{with} 5 \% \mathrm{CO}_{2}$ for $6 \mathrm{~h}$. The medium was replaced and they were transfected for $48 \mathrm{~h}$. Ultimately, qRT-PCR was employed to measure transfection efficiency.

\section{Luciferase reporter assay}

To verify the targeting relationship between circ_0048856 and miR-1287-5p, we performed a luciferase reporter analysis. Circ_0048856-WT or circ_0048856-MUT (100 ng) were co-transfected with $100 \mathrm{nM}$ miR1287-5p mimics or miR-1287-5p mimics NC into NSCLC cells using Lipofectamine 2000 (Invitrogen, CA, USA) following the instruction of manufacture. 48 hours after transfection, the cells were harvested and detected by Dual-Luciferase Reporter Assay System Kit (Promega, Madison, WI, USA). The specific operation was carried out according to the instructions.

\section{Statistical analysis}

We used the software of SPSS 18.0 (SPSS. Chicago. IL, USA) was performed to analyze all data, and all graphs were plotted via GraphPad Prism (GraphPad, San Diego, CA, USA). The correlation between circ_0048856 expression and clinicopathological features was assessed via $\chi^{2}$ test. Difference between groups was compared by Student's t-test. Kaplan-Meier and cox regression analyses were conducted to assess the prognostic ability of circ_0048856 in NSCLC. $P<0.05$ was seen as obviously significant difference.

\section{Results}


Expression of circ_0048856 is up-regulated in the serum of NSCLC patients

The relative expression of Circ_0048856 in serum samples of NSCLC subjects and healthy individuals were measred via qRT-PCR. All data revealed that the expression of circ_0048856 in NSCLC serum samples was significantly up-regulated than that in healthy volunteers' serum specimens $(P<0.001$, Figure 1).

\section{Relationship between circ_0048856 expression and clinical features}

The patients diagnosed with NSCLC were divided into low expression group $(n=58)$ and high expression group ( $n=63)$, according to their median serum levels of circ_0048856. In addition, we examined the relationship between circ_0048856 levels and the clinical features of NSCLC patients using chi-square test. All data proved that circ_0048856 expression was significantly correlated with TNM stage $(P=0.002)$ and lymphatic node metastasis $(P<0.001)$. Nevertheless, circ_0048856 expression was not found to be associated with other clinical features including age, gender, smoking history, tumor size and differentiation (all $P>0.05$, Table 1).

\section{Prognostic value of circ_0048856 expression in patients with NSCLC}

To evaluate the prognostic ability of circ_0048856 for NSCLC cases, we assessed the connection between circ_0048856 levels and overall survival. The result of Kaplan-Meier with log rank test analysis revealed that the overall survival of patients with high circ_0048856 expression was obviously shorter than those with low circ_0048856 expression (log rank test, $P=0.002$, Figure 2).

Besides, we used the Cox regression analysis to assess the prognostic ability of circ_0048856 in NSCLC patients. Both univariable and multivariable analysis results listed in Table 2 showed that circ_0048856 expression ( $\mathrm{HR}=2.001,95 \% \mathrm{Cl}: 1.258-3.184, P=0.003$ and $\mathrm{HR}=1.669,95 \% \mathrm{Cl}: 1.018-2.736, P=0.042)$ and lymphatic node metastasis (HR=2.118 95\% Cl: 1.340-3.348, $P=0.001$ and $\mathrm{HR}=1.766,95 \% \mathrm{Cl}: 1.086-2.874$, $P=0.022$ ) were useful prognostic indicators for overall survival in cases with NSCLC.

Table 2. 


\begin{tabular}{|lllll|}
\hline Factors & \multicolumn{3}{c|}{ Univariate analysis } & \multicolumn{2}{c|}{ Multivariate analysis } \\
\cline { 2 - 5 } & $\mathrm{HR}(95 \% \mathrm{Cl})$ & $\boldsymbol{P}$ values & $\mathrm{HR}(95 \% \mathrm{Cl})$ & Pvalues \\
\hline circ_0048856 expression & $2.001(1.258-3.184)$ & 0.003 & $1.669(1.018-2.736)$ & 0.042 \\
\hline Age & - & 0.332 & - & - \\
\hline Gender & - & 0.999 & - & - \\
\hline Smoking history & - & 0.935 & - & - \\
\hline Tumor size & - & 0.666 & - & - \\
\hline TNM stage & - & 0.072 & - & - \\
\hline Lymph node metastasis & $2.118(1.340-3.348)$ & 0.001 & $1.766(1.086-2.874)$ & 0.022 \\
\hline Differentiation & - & 0.554 & - & - \\
\hline Note: - indicated no data. & & & & \\
\hline
\end{tabular}

\section{Circ_0048856 directly targeted $m i R-1287-5 p$}

We predicted that miR-1287-5p might be the direct downstream miRNA of circ_0048856 through online software Starbase v2.0 (Figure 3A). To further verify the relationship between circ_0048856 and miR1287-5p was evaluated by luciferase reporter assay. Circ_0048856-3'UTR-WT or circ_0048856-3'-UTRMUT were co-transfected with $100 \mathrm{nM}$ miR-1287-5p mimics or mimics NC into A549 and H1299 cells using Lipofectamine 2000 following the instruction of manufacture. The data revealed that in the cells transfected with circ_0048856-3'UTR-WT, miR-1287-5p mimics had significantly lower luciferase activity; when the binding site was mutated, the above inhibition was lost (Figure 3B and C). The experiment shows that miR-1287-5p is the direct downstream miRNA of circ_0048856.

\section{Circ_0048856 has a negative correlation with $\operatorname{miR}-1287$}

To further explore the relationship between $m i R-1287-5 p$ and circ_0048856, we detected the expression level of miR-1287-5p in serum of patients with NSCLC, and found that the expression level of miR-1287$5 p$ was significantly decreased (Figure 4A), which was contrary to the expression pattern of circ_0048856 in serum of patients with NSCLC. We also detected the expression of miR-1287-5p and circ_0048856, we found that the expression of miR-1287-5p in A549 and H1299 cells was lower than that in the control group, while the expression of circ_0048856 in A549 and H1299 cells was higher than that in the control group (Figure 4B). Furthermore, knockdown of circ_0048856 in NSCLC cells resulted in up regulation of miR-1287-5p expression (Figure 4C). Analysis of the correlation between circ_0048856 and miR-1287-5p mRNA expression in serum of NSCLC showed that there was a negative correlation between them. (Figure 4D). Our results proved that circ_0048856 is negatively correlated with miR-1287-5p.

\section{Discussion}


NSCLC is a kind of malignant tumor threatening the safety of human life, which has a high incidence and mortality. The disease is prone to metastasis and poor prognosis [18]. At present, many prognostic markers have been found, but the clinical application is less [19-21]. In this study, we confirmed that the expression of circ_0048856 in serum of NSCLC was increased, and the expression of circ_0048856 can be used as an independent prognostic biomarker for the overall survival rate of NSCLC patients, and it can also play a role by targeting $m i R-1287-5 p$.

In recent years, circRNAs have become a research hotspot. The role of circRNAs is to isolate or "sponge" other gene expression regulators, especially miRNAs. CircRNAs play an important role in a variety of biological functions and have broad application prospects [22,23]. The accumulated data confirmed that circRNAs may become a new indicator for prognosis and diagnostic evaluation of lung cancer patients [24].

Previous studies have found that a large number of circRNAs are dysregulated in NSCLC. Although circRNAs are constantly found, their biological functions in NSCLC have just been explored [25-27]. We detected the expression level of circ_0048856 in serum of NSCLC by qRT-PCR, and found that the expression level of circ_0048856 was significantly up-regulated, which was consistent with previous studies [17]. The high expression of circ_0048856 was significantly correlated with TNM stage and lymph node metastasis. Survival analysis showed that the overall survival of patients with high expression of circ_0048856 was significantly shorter than that of patients with low expression of circ_0048856. Further analysis showed that circ_0048856 expression and lymph node metastasis were independent prognostic biomarkers of overall survival in NSCLC patients. A study by Wang et al. found that circ_0067934 was upregulated in NSCLC tissues and cells, and its high expression was closely related to TNM stage, lymph node status and distant metastasis, indicating poor prognosis of NSCLC [28]. Geng et al. reported that the expression of circ-MTHFD2 was up-regulated in NSCLC samples, and its high expression indicates poor prognosis of patients, which can be used as a diagnostic, prognostic marker and therapeutic target of NSCLC patients [29]. Liu et al. confirmed that the low expression of circ_0001649 is associated with poor prognosis of NSCLC patients, and can be used as a prognostic marker of NSCLC [30].

In addition, we predicted the direct related miRNAs of circ_0048856 by online prediction software Starbase V2.0, and found that miR-1287-5p may be the direct downstream miRNA of circ_0048856. Furthermore, we confirmed that miR-1287-5p is a direct downstream miRNA of circ_0048856 by luciferase reporter gene analysis. Moreover, the expression of miR-1287-5p was significantly down regulated in NSCLC serum and cell lines, and the expression trend was opposite to circ_0048856, showing a negative correlation. We speculate that circ_0048856 is involved in the progression of NSCLC by targeting miR-1287-5p.

Previous studies have shown that circRNAs can play a role in NSCLC by targeting miRNAs. Li et al. identified a new circRNA, circ_0002483, which can sponge multiple miRNAs and inhibit the progression of NSCLC through sponge miR-182-5p [31]. Another study found that circ_0000735 had a targeting relationship with miR-1179/1182. Circ_0000735 combined with miR-1179/1182 and participated in the 
biological process of NSCLC cells [32]. Gao et al. confirmed that circ_0074027 is carcinogenic in NSCLC, and its high expression indicates poor prognosis. Circ_0074027 can promote the process of NSCLC cells as competitive endogenous RNA through miR-185-3p/BRD4/MADD pathway [33]. These studies have confirmed that circRNAs has a significant biological function in NSCLC, and can be used as a target for the diagnosis, prognosis and treatment of NSCLC.

There are several limitations in this study that cannot be ignored. Firstly, we recruit fewer patients and volunteers, which may affect the accuracy of the results. Secondly, we did not explore the specific mechanism of circ_0048856 in NSCLC. The pathogenesis of NSCLC is very complex. In the next step, we will expand the sample size and further explore the molecular mechanism of circ_0048856 in the occurrence and development of NSCLC, so as to bring new hope for the treatment of patients.

\section{Conclusion}

To sum up, circ_0048856 may be an independent prognostic biomarker in patients with NSCLC. There is a negative correlation between circ_0048856 and miR-1287-5p. circ_0048856 may participate in the occurrence and development of NSCLC by targeting miR-1287-5p. Our study provides a new target for the treatment and prognosis of the disease, and provides a new theoretical basis for the prevention and treatment of the disease.

\section{Abbreviations}

Non small cell lung cancer (NSCLC)

Small cell lung cancer (SCLC)

Circular RNAs (circRNAs)

MicroRNA (miRNA)

Human bronchial epithelial cell (HBE)

American Type Culture Collection (ATCC)

Quantitative real-time PCR (qRT-PCR)

\section{Declarations}

\section{Ethics approval and consent to participate}

This study was supported by the Ethics Committee of Inner Mongolia People's Hospital and also has been carried out in accordance with the World Medical Association Declaration of Helsinki. 
The subjects had been informed the objective. Certainly, written consents were signed by every subject in this study.

\section{Consent for publication}

We obtaining permission from participants to publish their data.

\section{Availability of data and materials}

All data generated or analysed during this study are included in this published article.

\section{Competing interests}

The authors declare that they have no competing interests.

\section{Funding}

Not applicable.

\section{Authors' contributions}

Y.H. and W.L. design of the work; Y.L. the acquisition, analysis, N.C. interpretation of data; Y.G. and F.L. the creation of new software used in the work; M.Z. have drafted the work or substantively revised it. All authors read and approved the final manuscript.

\section{Acknowledgements}

Not applicable.

\section{References}

1. de Sousa VML, Carvalho L: Heterogeneity in Lung Cancer. Pathobiology : journal of immunopathology, molecular and cellular biology 2018, 85(1-2):96-107.

2. Hirsch FR, Scagliotti GV, Mulshine JL, Kwon R, Curran WJ, Jr., Wu YL, Paz-Ares L: Lung cancer: current therapies and new targeted treatments. Lancet 2017, 389(10066):299-311.

3. Howlader N, Forjaz G, Mooradian MJ, Meza R, Kong CY, Cronin KA, Mariotto AB, Lowy DR, Feuer EJ: The Effect of Advances in Lung-Cancer Treatment on Population Mortality. The New England journal of medicine 2020, 383(7):640-649.

4. Schabath MB, Cote ML: Cancer Progress and Priorities: Lung Cancer. Cancer epidemiology, biomarkers \& prevention : a publication of the American Association for Cancer Research, cosponsored by the American Society of Preventive Oncology 2019, 28(10):1563-1579.

5. Bade BC, Dela Cruz CS: Lung Cancer 2020: Epidemiology, Etiology, and Prevention. Clinics in chest medicine 2020, 41(1):1-24. 
6. Hoy H, Lynch T, Beck M: Surgical Treatment of Lung Cancer. Critical care nursing clinics of North America 2019, 31(3):303-313.

7. Duma N, Santana-Davila R, Molina JR: Non-Small Cell Lung Cancer: Epidemiology, Screening, Diagnosis, and Treatment. Mayo Clinic proceedings 2019, 94(8):1623-1640.

8. Shroff GS, Viswanathan C, Carter BW, Benveniste MF, Truong MT, Sabloff BS: Staging Lung Cancer: Metastasis. Radiologic clinics of North America 2018, 56(3):411-418.

9. Diallo LH, Tatin F, David F, Godet AC, Zamora A, Prats AC, Garmy-Susini B, Lacazette E: How are circRNAs translated by non-canonical initiation mechanisms? Biochimie 2019, 164:45-52.

10. Patop IL, Wust S, Kadener S: Past, present, and future of circRNAs. The EMBO journa/ 2019, 38(16):e100836.

11. Cao YZ, Sun JY, Chen YX, Wen CC, Wei L: The roles of circRNAs in cancers: Perspectives from molecular functions. Gene 2021, 767:145182.

12. Zhang Q, Wang W, Zhou Q, Chen C, Yuan W, Liu J, Li X, Sun Z: Roles of circRNAs in the tumour microenvironment. Molecular cancer 2020, 19(1):14.

13. Harper KL, McDonnell E, Whitehouse A: CircRNAs: From anonymity to novel regulators of gene expression in cancer (Review). International journal of oncology 2019, 55(6):1183-1193.

14. Geng Y, Jiang J, Wu C: Function and clinical significance of circRNAs in solid tumors. Journal of hematology \& oncology 2018, 11(1):98.

15. Xu P, Wang L, Xie X, Hu F, Yang Q, Hu R, Jiang L, Ding F, Mei J, Liu J et al: Hsa_circ_0001869 promotes NSCLC progression via sponging miR-638 and enhancing FOSL2 expression. Aging 2020, 12(23):23836-23848.

16. Sun H, Chen Y, Fang YY, Cui TY, Qiao X, Jiang CY, Lu ZB: Circ_0000376 enhances the proliferation, metastasis, and chemoresistance of NSCLC cells via repressing miR-384. Cancer biomarkers : section A of Disease markers 2020, 29(4):463-473.

17. Chen T, Luo J, Gu Y, Huang J, Luo Q, Yang Y: Comprehensive analysis of circular RNA profiling in AZD9291-resistant non-small cell lung cancer cell lines. Thoracic cancer 2019, 10(4):930-941.

18. Bica-Pop C, Cojocneanu-Petric R, Magdo L, Raduly L, Gulei D, Berindan-Neagoe I: Overview upon miR21 in lung cancer: focus on NSCLC. Cellular and molecular life sciences : CMLS 2018, 75(19):35393551.

19. Incorvaia L, Fanale D, Badalamenti G, Barraco N, Bono M, Corsini LR, Galvano A, Gristina V, Listi A, Vieni S et al: Programmed Death Ligand 1 (PD-L1) as a Predictive Biomarker for Pembrolizumab Therapy in Patients with Advanced Non-Small-Cell Lung Cancer (NSCLC). Advances in therapy 2019, 36(10):2600-2617.

20. Piao L, Yang Z, Feng Y, Zhang C, Cui C, Xuan Y: LETM1 is a potential biomarker of prognosis in lung non-small cell carcinoma. BMC cancer 2019, 19(1):898.

21. Xie Y, Zhang Y, Du L, Jiang X, Yan S, Duan W, Li J, Zhan Y, Wang L, Zhang S et al: Circulating long noncoding RNA act as potential novel biomarkers for diagnosis and prognosis of non-small cell lung 
cancer. Molecular oncology 2018, 12(5):648-658.

22. Arnaiz E, Sole C, Manterola L, Iparraguirre L, Otaegui D, Lawrie CH: CircRNAs and cancer: Biomarkers and master regulators. Seminars in cancer biology 2019, 58:90-99.

23. Lei M, Zheng G, Ning Q, Zheng J, Dong D: Translation and functional roles of circular RNAs in human cancer. Molecular cancer 2020, 19(1):30.

24. Huang X, Zhang W, Shao Z: Prognostic and diagnostic significance of circRNAs expression in lung cancer. Journal of cellular physiology 2019, 234(10):18459-18465.

25. Huang MS, Liu JY, Xia XB, Liu YZ, Li X, Yin JY, Peng JB, Wu L, Zhang W, Zhou HH et al: Hsa_circ_0001946 Inhibits Lung Cancer Progression and Mediates Cisplatin Sensitivity in Non-small Cell Lung Cancer via the Nucleotide Excision Repair Signaling Pathway. Frontiers in oncology 2019, 9:508.

26. Zheng JP, Dai YM, Chen Z, Chen Q, Zheng Y, Lin X, Cui TJ: Circular RNA circ-ABCB10 promotes nonsmall cell lung cancer proliferation and inhibits cell apoptosis through repressing KISS1. European review for medical and pharmacological sciences 2020, 24(5):2518-2524.

27. Tan Z, Cao F, Jia B, Xia L: Circ_0072088 promotes the development of non-small cell lung cancer via the miR-377-5p/NOVA2 axis. Thoracic cancer 2020, 11(8):2224-2236.

28. Wang J, Li H: CircRNA circ_0067934 silencing inhibits the proliferation, migration and invasion of NSCLC cells and correlates with unfavorable prognosis in NSCLC. European review for medical and pharmacological sciences 2018, 22(10):3053-3060.

29. Geng QQ, Wu QF, Zhang Y, Zhang GJ, Fu JK, Chen NZ: Clinical significance of circ-MTHFD2 in diagnosis, pathological staging and prognosis of NSCLC. European review for medical and pharmacological sciences 2020, 24(18):9473-9479.

30. Liu T, Song Z, Gai Y: Circular RNA circ_0001649 acts as a prognostic biomarker and inhibits NSCLC progression via sponging miR-331-3p and miR-338-5p. Biochemical and biophysical research communications 2018, 503(3):1503-1509.

31. Li X, Yang B, Ren H, Xiao T, Zhang L, Li L, Li M, Wang X, Zhou H, Zhang W: Hsa_circ_0002483 inhibited the progression and enhanced the Taxol sensitivity of non-small cell lung cancer by targeting miR-182-5p. Cell death \& disease 2019, 10(12):953.

32. Li W, Jiang W, Liu T, Lv J, Guan J: Enhanced expression of circ_0000735 forecasts clinical severity in NSCLC and promotes cell progression via sponging miR-1179 and miR-1182. Biochemical and biophysical research communications 2019, 510(3):467-471.

33. Gao P, Wang Z, Hu Z, Jiao X, Yao Y: Circular RNA circ_0074027 indicates a poor prognosis for NSCLC patients and modulates cell proliferation, apoptosis, and invasion via miR-185-3p mediated BRD4/MADD activation. Journal of cellular biochemistry 2020, 121(3):2632-2642.

\section{Figures}




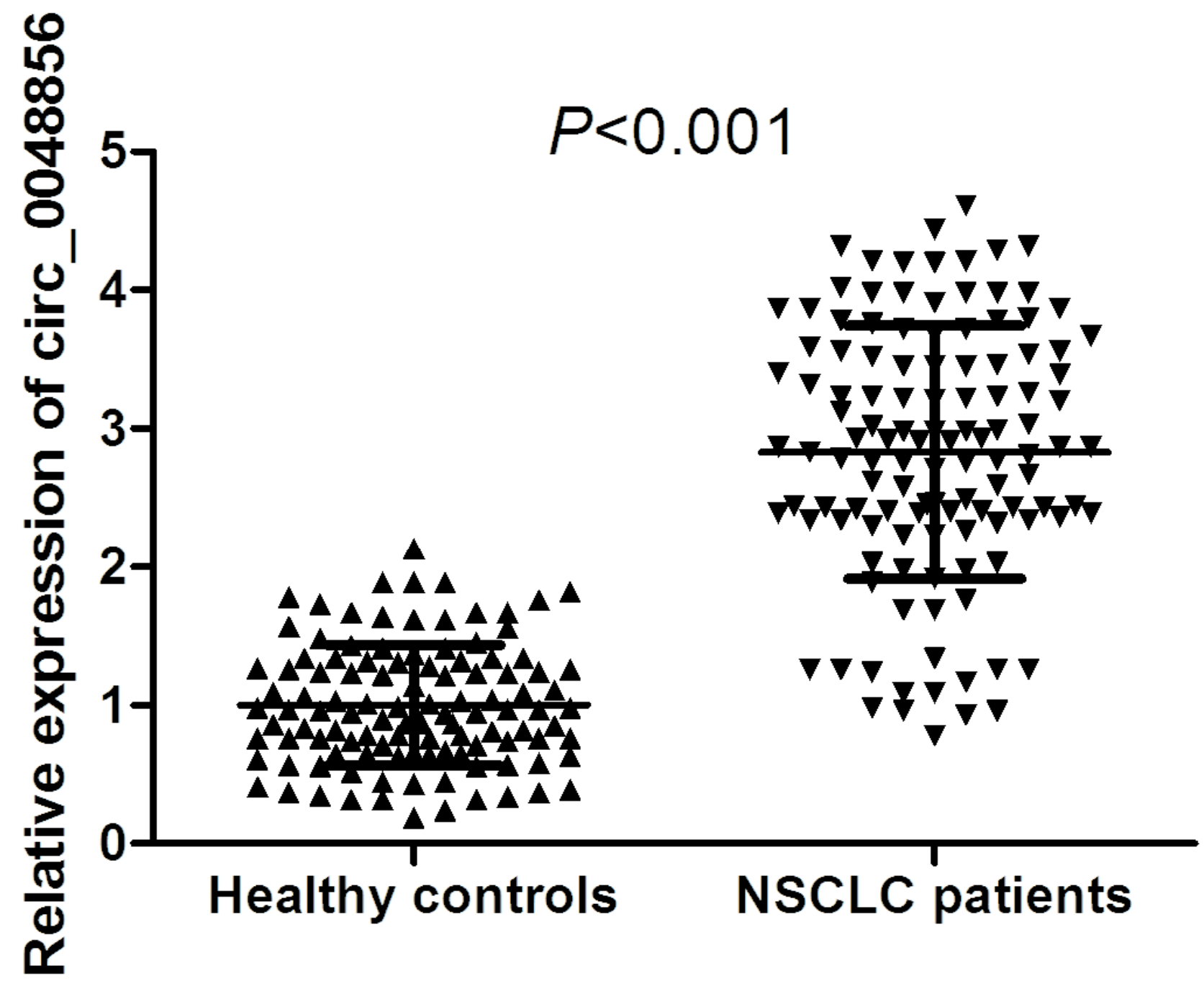

Figure 1

Serum circ_0048856 expression levels detected by qRT-PCR in NSCLC patients and healthy controls. The results showed that the expression of circ_0048856 was significantly increased in NSCLC patients. $\mathrm{P}<0.001$. 


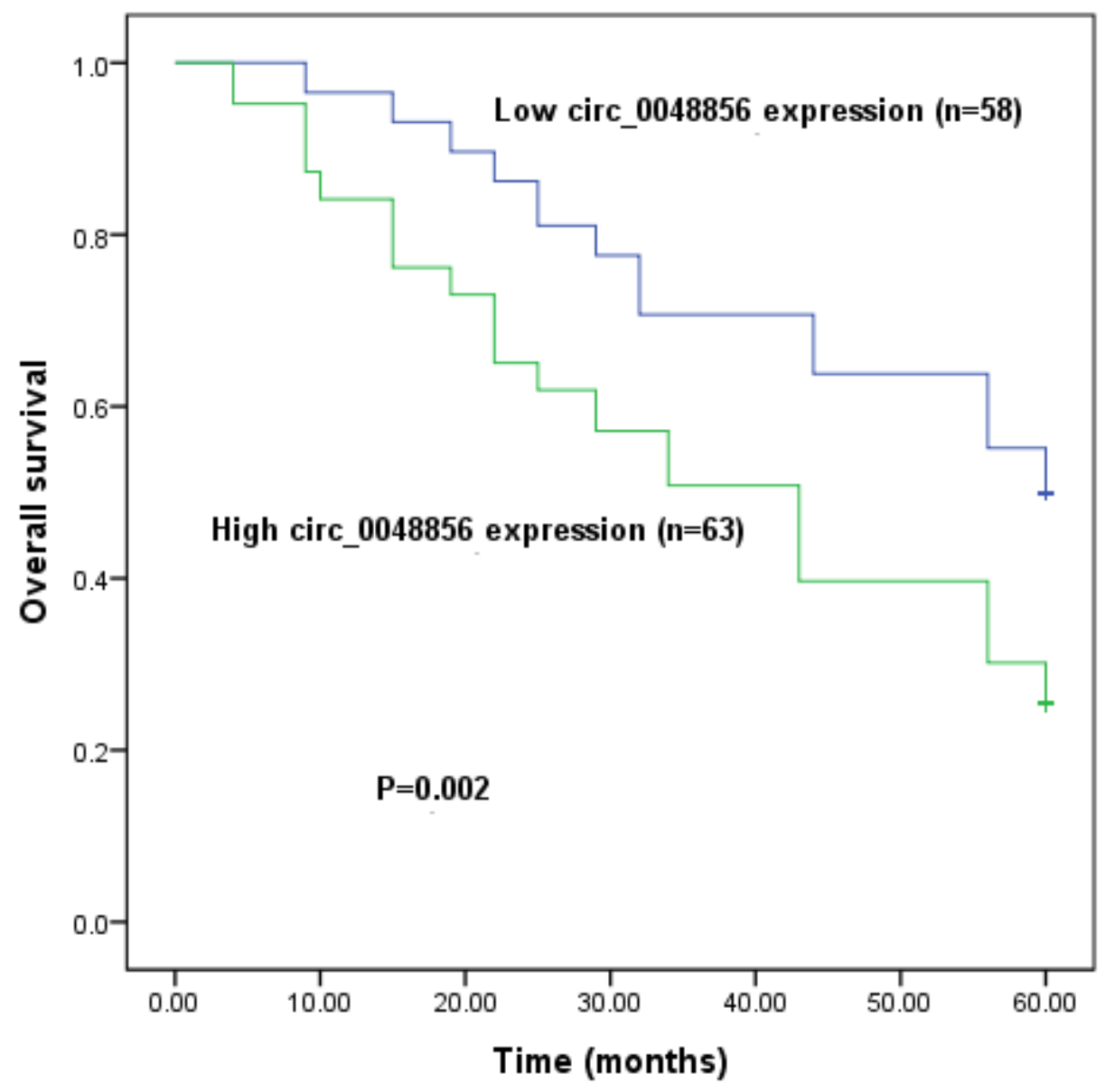

Figure 2

Survival analysis. The overall survival of patients with high circ_0048856 expression was significantly shorter than those with low circ_0048856 expression (log rank test, $\mathrm{P}=0.002$ ). 
A

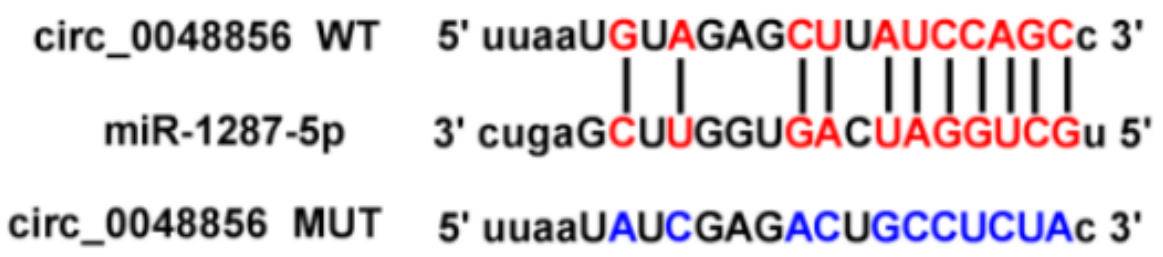

B

A549

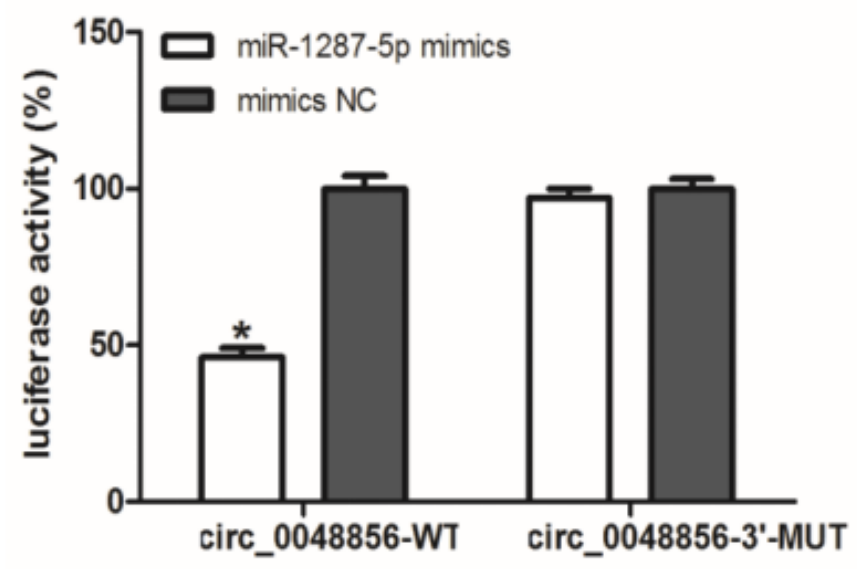

H1299

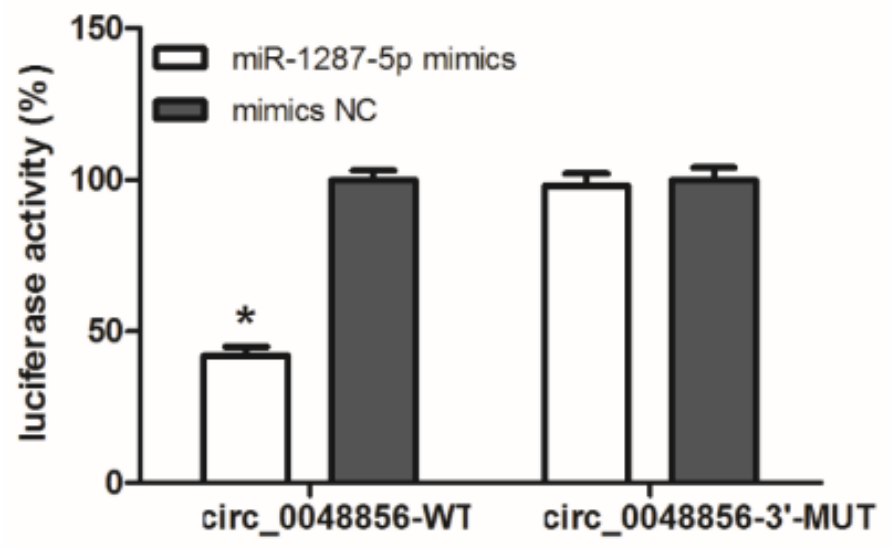

Figure 3

Circ_0048856 directly targetedmiR-1287-5p. A, The direct target relationship predicted by starBase v2.0 between circ_0048856 and miR-1287-5p. B and C, Luciferase reporter assay to verify the targeting relationship between circ_0048856 and miR-1287-5p. * $P<0.05$. 

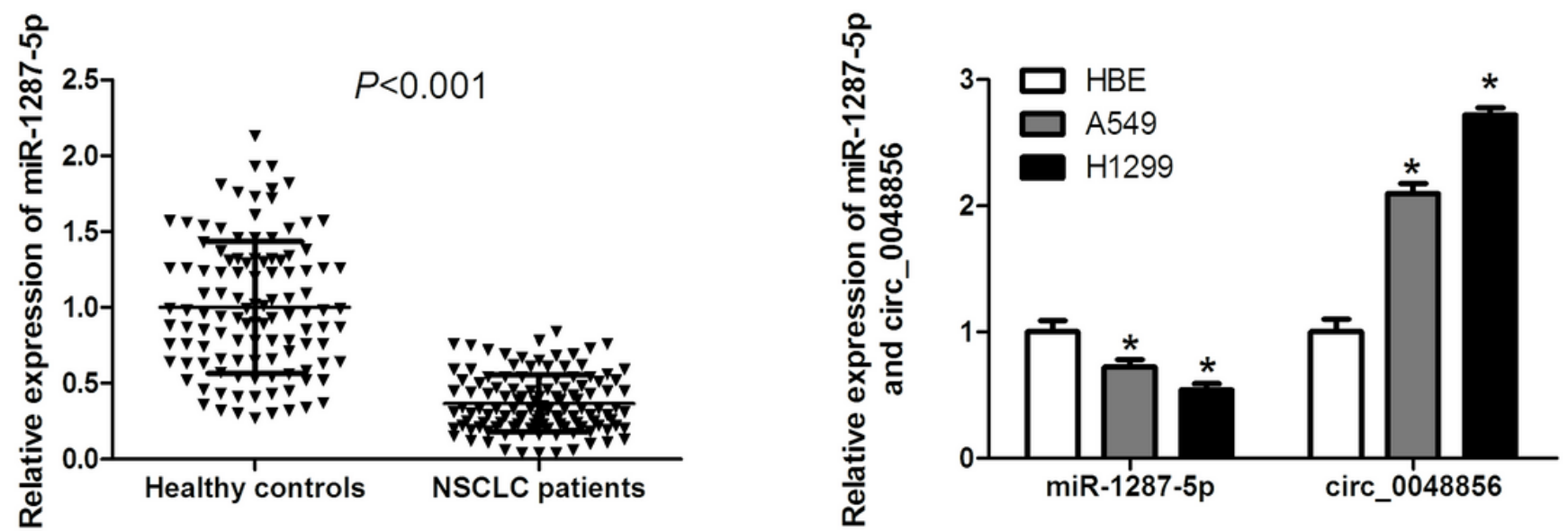

C
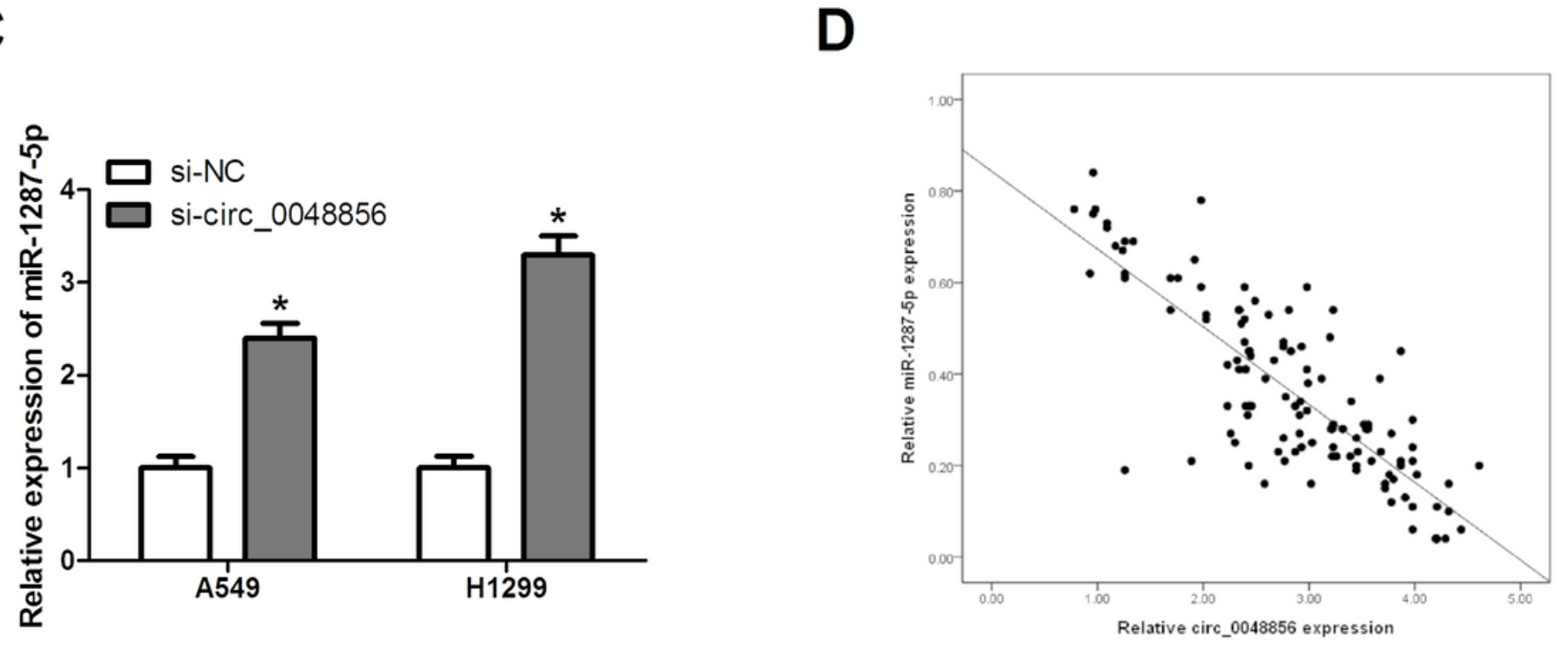

Figure 4

Circ_0048856 was negatively correlated with miR-1287-5p. A, Expression of miR-1287-5p in serum of NSCLC, B, Expression of circ_0048856 and miR-1287-5p in NSCLC cell lines, C, Knockdown of circ_0048856 in NSCLC cells, miR-1287-5p expression was up-regulated, D, Correlation analysis of circ_0048856 and miR-1287-5p mRNA expression in serum of NSCLC. ${ }^{*} P<0.05$. 